\title{
„Was ist Migration?“ Risiken eines essentialistischen Migrationsbegriffs in der Erwachsenenbildung
}

\author{
Malte Ebner von Eschenbach
}

Eingegangen: 15. November 2015 / Angenommen: 1. März 2016 / Online publiziert: 3. Mai 2016

(C) Die Autoren 2016. Dieser Artikel ist auf SpringerLink mit Open Access verfügbar

Zusammenfassung Auch wenn die Appelle, die Bedeutung von Migration für Erwachsenenbildung deutlicher wahrzunehmen, unüberhörbar sind, bleiben sie bezüglich kategorialer Arbeit bemerkenswert wenig beachtet. Grundlagentheoretisch motivierte Arbeit am Begriff „Migration“ ist in der Erwachsenenbildung noch lange nicht hinreichend ausgeschöpft. Auch wenn sich einzelne Studien mit ihm auseinandersetzen, besteht dennoch der Eindruck, dass kategoriale Klärungsversuche singulär bleiben. Die nicht einfache Aufgabe, den Begriff Migration vor seiner kategorialen Stilllegung zu bewahren, bleibt eine ernsthafte Herausforderung für erwachsenenpädagogische Migrationsforschung, sofern sie daran interessiert ist, die Risiken eines bisher essentialistischen Kurses ernsthaft ins Visier zu nehmen.

Schlüsselwörter Migration · Essentialismus · Kontingenz

\begin{abstract}
While the calls to take more notice of the significance of migration for adult education are loud and clear, notably little attention is paid to them with regard to work on categories. In adult education, fundamental theoretical work on definitions of "migration" is still far from complete. Even though individual studies deal with the term, it seems that attempts to clarify categories remain scarce. If adult education research on migration has a genuine interest in examining the risks of the hitherto essentialist course, the none-too simple task of preserving the term "migration" from categorial closedown remains a serious challenge.
\end{abstract}

Keywords Migration · Essentialism · Contingency perspective

M. Ebner von Eschenbach $(\bowtie)$

Universität Potsdam, Potsdam, Deutschland

E-Mail: malte.ebner.von.eschenbach@uni-potsdam.de 


\section{Einleitung}

Eine kritische Reflexion der stillschweigenden Voraussetzungen des Migrationsbegriffs steht in der Erwachsenenbildung noch am Anfang. Auch wenn die Appelle, die Relevanz von Migration für Erwachsenenbildung wahrzunehmen, unüberhörbar sind, bleiben sie bezüglich kategorialer Arbeit bemerkenswert wenig beachtet. Obschon Migration in vielerlei Hinsicht gesellschaftlich präsent ist - nicht nur aufgrund der aktuell dramatischen Ereignisse (vgl. Heimeshoff et al. 2014; Kursbuch 2015) - scheint Migration in disziplinären Zusammenhängen der Erwachsenenbildung grundsätzlich noch zu wenig Aufmerksamkeit zu erhalten. So konstatiert Nuissl (2012) im Editorial des Report zum Thema „Bildung und Migration“, dass kaum Beiträge für diese geplante Ausgabe zu verzeichnen waren, und dass von den Einreichungen mehr als die Hälfte nach dem Review-Verfahren abgelehnt werden musste. Diese „Diskrepanz zwischen der Realität einer Einwanderungsgesellschaft und ihrer wissenschaftlichen Bearbeitung zu erklären“ (ebd., S. 7), falle nicht leicht. Eine Erklärung läge evtl. darin, dass vielleicht, ,einfach die grundlegenden Fragen zur Migration in Deutschland mittlerweile erforscht“ (ebd.) seien. Nuissls Äußerung eröffnet eine Deutung, in der geradezu der Mangel grundlagentheoretischer Arbeit hervorgehoben wird. Auch wenn die „Realität einer Einwanderungsgesellschaft““ nicht mehr geleugnet wird, die geringe wissenschaftliche Aufmerksamkeit bleibt weiterhin beklagenswert. Beispielsweise formulierte Heinemann in ihrer Dissertation Teilnahme an Weiterbildung in der Migrationsgesellschaft demgemäß, dass ,eine erhöhte Aufmerksamkeit der Erwachsenenbildungsforschung für migrationsspezifische Themen dringend notwendig (ist)“ (Heinemann 2014, S. 35). In ähnlicher Tonlage legt Öztürk im Studientext Migration und Erwachsenenbildung dar, dass seine Ziele darin bestehen, ,eine fundierte Wissens- und Reflexionsgrundlage der Migrationsthematik in der Weiterbildung zu schaffen“ (Öztürk 2014, S. 9) und „Herausforderungen [zu] identifizieren, die sich für die Weiterbildung durch Migration bzw. migrationsbedingte Vielfalt ergeben“ (ebd., S. 10). Öztürks Hinweis auf die bisherige Ermangelung einer „fundierten Wissens- und Reflexionsgrundlage“ unterstützt nicht nur Heinemanns Aussage, sondern wiederholt Sprungs Feststellung, dass zu Migration in der Erwachsenenbildung ,,[nach wie vor] empirische Forschungen sowie theoretische Reflexionen (...) nur im geringen Umfang vorlägen“ (Sprung 2008, Kap. 2). Im Spektrum jüngerer Veröffentlichungen zu migrationsspezifischen Themen in der Erwachsenenbildung wird zwar ein leichter Aufmerksamkeitsanstieg erkennbar, obwohl dieser nicht grundsätzlich über die gegenwärtige Situation hinwegtäuschen sollte (vgl. u. a. Hamburger 2010; Bilger und Hartmann 2011; Fischer 2011; Sprung 2011, 2015; Heinemann und Robak 2012; Heinemann 2014; Öztürk 2014).

Programmatische Appelle, die die Bedeutung von Migration für Erwachsenenbildung hervorheben und deren Forderung lautet, sich dem Phänomen stärker zu widmen, tauchen weiterhin auf (vgl. z. B. Schrader 2015). Derlei emphatische Forderungen verstummen nicht und weisen in wiederkehrenden Formulierungen auf die marginale Rolle migrationsspezifischer Themen in der Erwachsenenbildung hin, wobei jedoch kategoriale Arbeit geradezu ausgeklammert zu sein scheint (vgl. einige Ausnahmen - z. B. Mecheril et al. 2013; Heinemann 2014, S. 17 ff.). Damit 
entsteht der Eindruck, es sei bereits im Vorfeld migrationsspezifischer Forschung in der Erwachsenenbildung hinreichend geklärt, was unter Migration oder unter Migrant_innen zu verstehen sei und welche Charakteristika sie auszeichnen. Zudem fällt auf, dass gerade quantitativ-empirische Studien Migration überwiegend vor dem Hintergrund von „beschäftigungspolitischen Imperativen“ (Hamburger 2010, S. 887) thematisieren (vgl. u. a. Bilger 2006; Schmidt und Tippelt 2006; Bilger 2011; Öztürk 2012; Sprung 2015). Gleichwohl wird vonseiten quantitativ-empirischer Forschung signalisiert, dass Folgendes weiterhin gelte: „Personen mit Migrationshintergrund sind eine weitgehend unbekannte Zielgruppe in der Weiterbildung" (Bilger 2011, S. 353). Letztlich deutet die beunruhigende Gesamtsituation auf eine (implizite) „Stilllegung der Kategorie“ Migration in der Erwachsenenbildung.

Die affirmative Haltung gegenüber einem weiterhin ungeklärten Verständnis von Migration soll hier als ,kategoriale Stilllegung“ bezeichnet werden und im folgenden Argumentationsgang nachgezeichnet werden. Entlang einer begrifflichen Annäherung werden erhebliche Zweifel an der unausgesprochenen Unterstellung angemeldet, Migration würde sich auf ein eindeutiges geschlossenes Kollektiv beziehen, was sich anhand spezifischer Eigenschaften markieren ließe. Hierin drückt sich bereits das Misstrauen gegenüber essentialistisch gefassten Forschungsgegenständen aus, deren gesellschaftliche Folgen gewaltsam wirken. Die bisher ausbleibende Thematisierung der präempirischen Voraussetzungen des Migrationsbegriffs verhindert es geradezu, ,grundlegende Fragen zur Migration“ (Nuissl 2012, S. 7) in Angriff zu nehmen und epistemischen Protest gegen diese Unterlassung zu mobilisieren. Unter „präempirisch“" werden in diesem Zusammenhang vorgängige Deutungsmuster verstanden, die der Gegenstandsbestimmung und der wissenschaftlichen Erfahrbarkeit implizit zugrunde liegen und aufgrund ihrer Axiomatik empirisch weder verifizierbar noch widerlegbar sind (vgl. Sandkühler 1990). Auf eben dieser scheinbaren Neutralität des „Faktischen“ beruht ihre gesellschaftspolitische Brisanz.

\section{Migration begrifflich ,aus den Angeln heben““}

Mit der Skepsis gegenüber essentialistischen Zugängen zu Migration wird davon ausgegangen, dass entscheidende und kaum rückführbare gesellschaftspolitische Vorentscheidungen bereits auf kategorialer Ebene getroffen werden. Das jeweils gewählte „epistemologische Profil“ (Bachelard 1980) ermöglicht und begrenzt die Erfassung des Phänomens, weshalb dessen Einfluss auf grundlagentheoretische Fragestellungen folgenreich ist. Bereits der oftmals nicht wahrgenommene und zunächst unverdächtig erscheinende Akt der Gegenstandsbestimmung mit seiner begrifflichen „Zuschneidung“ (vgl. Deleuze und Guattari 2000, S. 24) nimmt verdeckt Einschließungen und Ausschließungen vor. Demzufolge werden Begriffe als ,politische Kampfbegriffe" (Lichtblau 2011, S. 12) sichtbar, deren präempirische Rahmungen Aufmerksamkeit benötigen, weil sie „Ergebnis zielgerichteter Verfahren der Macht [sind]" (Butler 2010, S. 9). Die Rahmungen (frames) entscheiden zwar nicht allein, welche Aspekte sichtbar bzw. unsichtbar sind. Jedoch begrenzen sie die „Sphäre des Erscheinens" (ebd.). Sie setzen eine Unterscheidung voraus, die ohne kategoriale Reflexion unsichtbar bliebe. In diesem Sinne ist das Politische begrifflicher Arbeit 
verankert (vgl. Bedorf und Röttgers 2010) und die Bedeutung epistemischer Widerständigkeit unabweisbar. Insofern beschreibt Arbeit am Begriff ,nicht abstrakte Spekulation, sondern eine praktische" (Marchart 2013, S. 425) und stellt sich zugleich der künstlichen Trennung von Theorie und Praxis entgegen - Theorie ist gleich Praxis. Diese kontextsensible Fokussierung der Konstitutionsbedingungen des Migrationsbegriffs scheint in ihrem Potenzial für Erwachsenenbildung noch nicht ausgeschöpft.

In der Auseinandersetzung mit der Frage, was denn nun Migration „eigentlich“ kennzeichnet, bzw. wer „Migrant_innen“ oder „Personen oder Menschen mit Migrationshintergrund“" seien, werden die Folgen jeder definitorischen Rahmung deutlich, ermöglichen und verunmöglichen sie doch ein je spezifisches Sprechen oder Schweigen über Migration. Um die kategorialen Verankerungen dieser Deutungen sichtbar machen zu können, erscheint es zunächst notwendig, sich dem gegenwärtigen Hintergrund von Migration zuzuwenden.

Grob vereinfacht lassen sich die Diskurse zu Migration vor dem Hintergrund dreier sich wechselseitig verstärkender und ineinander verschiebender frames aufspannen:

(a) Zunächst wird Migration mit Diskursen zu Inklusion und Integration eng gekoppelt (vgl. Karakayali und Tsianos 2007; Mecheril 2011; Sprung 2012, S. 17; Castro Varela 2013a). Diese Debatten thematisieren Migration vornehmlich unter dem Gesichtspunkt einseitiger Anpassung oder „Eingliederung der Migranten in die Aufnahmegesellschaft" (Han 2006, S. 149). Zudem schwingen die Bedrohung des Wohlfahrtsstaates und „Fragmentierung“ der „Aufnahmegesellschaft“ als pejorative Konnotationen mit (vgl. Mecheril et al. 2013, S. 16). Die Dynamik dieses „Integrationsdispositivs“ (Mecheril 2011) ist darüber hinaus mit einem (b) sicherheitspolitischen Denken verschachtelt. Die sog. securitization setzt einen Rahmen, der Migration ,als einen risikoreichen Akt versteht, der im Kontakt mit dem organisierten Verbrechen steht“ (Rocha 2014, S. 146). Im Rahmen dieser „Versicherheitlichung“ (Hess und Kasparek 2010, S. 16) werden Fragen zur Sicherheitspolitik mobilisiert und dominieren menschenrechts- oder sozialtheoretische Diskurse (vgl. Mbembe 2015, S. 50 f.). (c) Neben dem Integrations- und Sicherheitsdispositiv erhält das „Dispositiv der Kontrolle“ eine große Bedeutung in den Diskursen zu Migration. Das „Dispositiv der Kontrolle“ (Buckel et al. 2014, S. 16) ist dadurch gekennzeichnet, dass migratorische Mobilität nicht grundsätzlich politisch-administrativ unterbrochen wird, sondern dass ,risikobehaftete“ Gruppen in ihrer Mobilität behindert bzw. eingeschränkt werden. Nicht nur außerhalb, sondern auch innerhalb Europas wird dementsprechend ,das ,Sichere“ vom ,Gefährlichen“ zu unterscheiden [versucht], ohne den (positiven) Zirkulationsfluss übermäßig zu behindern“ (Herrmann 2014, S. 162). Hieran knüpft die Unterscheidung zwischen erwünschter und unerwünschter Mobilität an, weshalb Mobilität gerade dann positiv bewertet, ermöglicht und gefördert wird, sobald sie volkswirtschaftlich nützlich erscheint (vgl. Zetter 2015, S. 55). Die damit gegenwärtig sichtbar werdende „Krise der Politik“ (ebd., S. 43) - die vonseiten politisch-administrativer Mandatsträger_innen noch als vermeintliche „Flüchtlings-“ oder „Einwanderungskrise“ umzudeuten versucht wird - zeigt, wie sich krampfhaft gegen die bereits eingetretene Obsoleszenz des territo- 
rialen Nationalstaatsparadigmas zur Wehr gesetzt wird (vgl. zum „methodologischen Nationalismus“ Wimmer und Glick Schiller 2003).

Die skizzierten Rahmungen zeichnen sich dadurch aus, dass sie ihre Latenz zu wahren und somit ihre eigenen Konstruktionscharakteristika abzuschirmen versuchen. Für die Revisibilisierung dieser verschleierten Selbstverständlichkeitsstrukturen erhält die Einnahme einer Kontingenzperspektive eine entscheidende Rolle (vgl. Schäffter 2011).

Mit Kontingenz wird zunächst darauf verwiesen, dass erst durch die Sprengung ontologischer Verengungen überhaupt mögliche Alternativen sichtbar werden. Demnach ist Kontingenz an Konflikt gebunden, weil vermeintliche Alternativlosigkeit oder Eindeutigkeit radikal infrage gestellt wird und dadurch Auseinandersetzungen provoziert (vgl. Marchart 2013, S. 8). Kontingenz mobilisiert alternative Blickpunkte und bringt auch noch so selbstverständliche oder ,natürliche“ Ordnungen ins Wanken (vgl. Clam 2002, S. 23). Damit werden die „Bedingungen, die geschaffen wurden oder geschaffen werden müssen, um Gegenstände unter den jeweils zu bestimmenden Umständen zu Gegenständen empirischen Wissens zu machen“ (Rheinberger 2007, S. 11) überhaupt erst erkennbar. Mit der Fokussierung des sich verschiebenden Hintergrunds verändert sich die (vordergründige) Figur zwangsläufig in ihrer Bedeutung, selbst wenn sie in ihrer Gestalt unverändert erscheinen sollte.

Mit der Strategie der Kontingenzerhöhung und -revisibilisierung wird Migration begrifflich aus ,den Angeln“ zu heben versucht und alternativen Sichtweisen zugeführt, die dann (überhaupt erst) empirisch in den Blick geraten können. Mit einer solchen epistemischen Intervention, im weiteren Verlauf als ,epistemische Widerständigkeit" markiert, wird die (anscheinend bisher unproblematische) Verdinglichung von Migration und der produktive Charakter einer Kontingenzperspektive gegenüber traditionell eingeschliffenen Deutungsmustern erkennbar (vgl. Schäfer und Wimmer 2004).

Grundsätzlich wird mit epistemischer Widerständigkeit beabsichtigt, bisher selbstverständliche und engvertraute historisch-soziale Zusammenhänge vermeintlicher Grundlagen, die sich als unumstößliche ,natürliche“ Fundamente gebärden, in ihrer unüberschaubaren Ambiguität kontingenter Erfahrungsbereiche deutlich hervortreten $\mathrm{zu}$ lassen. Grundlegende Fundamente (foundations) werden massiv infrage gestellt, , besonders jene Positionen (...), die von fundamentalen, d. h. revisionsresistenten Prinzipien, Gesetzen oder objektiven Realitäten ausgehen, die jedem sozialen oder politischen Zugriff entzogen sind" (Marchart 2010, S. 15). Dabei geht es in dieser sog. postfundamental-orientierten Denkbewegung auch nicht darum, alle Grundlagen infrage zu stellen oder auch nur eine Position zu vertreten. In Anschluss an Butler besteht die „Aufgabe eher darin, zu fragen, was durch den theoretischen Schritt, Grundlagen festzulegen, autorisiert und was ausgeschlossen wird“" (Butler 1993, S. 37, Herv. i. O.). Die postfundamentale Orientierung epistemischer Widerständigkeit affirmiert geradezu Heterogenisierung und Pluralisierung „als ,Konstituens“ der Erziehungswissenschaft“ (Masschelein und Ricken 2002, S. 96). Damit unterstreicht epistemische Widerständigkeit ein prinzipielles Misstrauen gegenüber der Annahme unumstößlicher kategorialer Fundamente. Bliebe dieser Protestakt gegenüber kategorialen Setzungen aus, wirken diese unbeach- 
tet als „stille Norm“ (Dietze 2009, S. 25) fort. Die Anzweiflung fundamentaler Grundlagen und die Schwächung ihrer hegemonialen Dominanz verdichten demzufolge die politischen Implikationen epistemischer Widerständigkeit. In diesem Horizont schimmern fruchtbare Schnittstellen zwischen Erwachsenenbildung und Allgemeiner Erziehungswissenschaft durch (vgl. z. B. McLaren 1999; Masschelein und Ricken 2002; Masschelein 2003; Holzer 2009; Pongratz 2010; Erler et al. 2012; Klingovsky und Pawlewizc 2014; Bünger 2015; Schäffter 2015).

\section{Migration als Ausnahmezustand}

Sofern die Frage „, Was ist Migration?“ zur Bestimmung des Forschungsgegenstandes Migration angestimmt wird, zählt folgende Formulierung zum Inventar klassischer Auskünfte: „Unter dem Begriff der Migration [werden] allgemein solche Bewegungen von Personen und Personengruppen im Raum (...) verstanden, die einen dauerhaften Wohnortwechsel (...) bedingen“ (Han 2000, S. 7). In Anschluss an Treibel greift Öztürk eine ähnlich weit verbreitete und anerkannte Definition zu Migration auf: „Vereinfacht kann man ,Migration“ als einen Sammelbegriff für Wanderungsprozesse von Personen bezeichnen, die ,ihren Lebensmittelpunkt verlagern ' oder zu deren , altem Lebensmittelpunkt ein neuer hinzukommt' (Treibel 2008, S. 295)“ (Öztürk 2014, S. 12). In ihrem enzyklopädischen Eintrag Erwachsenenbildung im Migrationskontext zieht Fischer zur näheren Bestimmung von „Menschen mit Migrationshintergrund“ Daten des Statistischen Bundesamtes heran:

Zu den Menschen mit Migrationshintergrund zählen ,,alle nach 1949 auf das heutige Gebiet der Bundesrepublik Deutschland Zugewanderten, sowie alle in Deutschland geborenen Ausländer und alle in Deutschland als Deutsche Geborenen mit zumindest einem zugewanderten oder als Ausländer in Deutschland geborenen Elternteil“" (Statistisches Bundesamt 2010, S. 8; 2009 betrug ihr Anteil 16 Millionen, d. h. 19,6\%) (Fischer 2011, Fußnote 2).

Anschließend stellt sie jedoch fest, dass die „Migrantenbevölkerung keinen homogenen Block [bildet], auch wenn der Begriff Migrant eine gewisse Homogenität und Gemeinsamkeit an Lebenslagen suggeriert“ (ebd., S. 3) und mit ,ethno-nationalen Kategorien nicht angemessen erfasst werden kann" (ebd., S. 4; vgl. auch Mecheril et al. 2013). Ihr Vorschlag mündet in den Hinweis, die Ausdifferenzierung von Lebensstilen durch die Einführung von Milieustudien zu berücksichtigen.

Mit den versammelten Definitionen scheint es hinreichende Gewissheit über den kategorialen Status von Migration zu geben, um sie als sichere Basis für empirische Forschungsvorhaben zu verwenden, auch wenn neben Fischer auch Öztürk zu bedenken gibt, dass ,eine differenzierte und realitätsgerechte Bestimmung der Migration weiterhin eine Herausforderung für die Migrationsforschung [bleibt]“" (Öztürk 2014, S. 15). Vor etwa einer Dekade stellte Bilger ebenfalls fest, dass die „Datenlage zum Thema Weiterbildung und Migration (...) dürftig“ (Bilger 2006, S. 23) sei. Dies hänge u. a. mit der Schwierigkeit zusammen, „Personen mit Migrationshintergrund repräsentativ“ (ebd.) abbilden zu können, bzw. liege in der Herausforderung der „Messung der Variable ,Migrationshintergrund““ (ebd., S. 29). Daher 
schlägt Bilger vor, dass ,zur genaueren Darstellung des Migrationshintergrundes neben den üblichen demographischen Aspekten entsprechend die folgenden Merkmale (...) zu berücksichtigen [sind]: Staatsangehörigkeit, Geburtsland, Zuzugsjahr oder -alter, Geburtsland der Eltern, Umgangssprache in der Familie/Muttersprache“ (ebd., S. 23). Abschließend läuft ihr Resümee darauf hinaus, dass „,[m]it dem Anspruch, Personen mit Migrationshintergrund gut zu repräsentieren, zudem etwaige Sprachbarrieren bei der Erhebung weitestgehend ausgeschlossen werden [müssen]“ (ebd., S. 29; vgl. auch Bilger 2011; Öztürk 2014, S. 16 ff.; Öztürk und Reiter 2015, S. 31). Die erwähnten Herausforderungen der Gegenstandsbestimmung von Migration sind unübersehbar und werden mit einer Strategie der Ausdifferenzierung und Verfeinerung des Forschungsgegenstandes verfolgt. Auf der Suche nach einer „realitätsgerechten“ Bestimmung geraten variantenreich (immer wieder neue oder korrigierte) ontologische Merkmale in den Blick, die ihrerseits nach einer umfassenden und totalitären Kategorie streben. Ob die Verfolgung der feinen Unterschiede die grundsätzliche Problematik der von außen herangetragenen und durchgesetzten Bezeichnungs-, Zuschreibungs- und Herstellungspraktiken damit weiter im Blick hat oder ob nicht geradezu der Eindruck entsteht, dass die Ausdifferenzierung bereits Ausdruck einer weitaus sensibleren Form von Anrufungen eines (unterstellten) Kollektivs darstellt, bleibt fraglich.

Am Label „Migrationshintergrund“ verdeutlichen beispielsweise Hamburger und Stauf, dass das ,Etikett zum Stigma werden [kann], mit dem Personen oder Familien gebrandmarkt werden, als nicht dazugehörend. (...) Insofern reproduziert die Bezeichnung mit Migrationshintergrund eine Festschreibung und Trennung der Mehrheit von den Minderheiten, von wir und den Anderen" (Hamburger und Stauf 2009, S. 30, Herv. i. O.). In die gleiche Richtung argumentiert Castro Varela, denn die Bezeichnung ,,Menschen mit Migrationshintergrund“ verweist auf die Nichtzugehörigkeit einer großen Bevölkerungsgruppe und fixiert diese gewissermaßen“ (Castro Varela 2013a, S. 15) als unauflösbare anspruchsbegründende Identität. Für sie „[stellen] ,Menschen mit Migrationshintergrund“ eine Konzept-Metapher dar, die die Reinheit der Nation, des eigentlichen Volkes sichert und eine Exklusion im Namen der Nation immer möglich macht - immer androht“" (Castro Varela 2013b, S. 73). Hamburger und Stauf führen weiterhin aus, dass auch deshalb die „Bezeichnung mit Migrationshintergrund irreführend [ist], weil sie aus der Perspektive derer ohne Migrationshintergrund Bestimmbarkeit und Gemeinsamkeit einer Gruppe suggeriert" (Hamburger und Stauf 2009, S. 31) und dass diese (Zu-)Ordnung ,,(s)pätestens in der dritten Generation (...) [nichts] erklärt“ (ebd.). Aus diesen Gründen warnen Paul Mecheril et al. vor Migrationsforschung, die das ,Abstammungsdispositiv“ (Messerschmidt 2014, S. 10) mitführt, weil ,,[d]amit direkt oder indirekt die Realität gegebener, den Handlungsspielraum von Migrant_innen (etc.) konstituierender sozialer, politischer, kultureller und rechtlicher Verhältnisse [affirmiert wird]“ (Mecheril et al. 2013, S. 14).

Die essentialistische Herangehensweise an den Migrationsbegriff hat zur Folge, dass die auf Individuen bezogene Zuschreibungspraktik auf Kollektive wie Ethnien oder Nationalitäten hin typisiert werden. Mit der Verschleierung und Unterstellung einer „quasi-homogenen“ stabilen Gruppe wird es ermöglicht, eine asymmetrische Bedeutungsbezeichnung mit normativen Bewertungen zuzuordnen, die Kategorie 
mit einer Kette von Eigenschaften, die allen Gruppenmitgliedern gemeinsam seien, auszustatten und vor allem mit wissenschaftlicher Forschung erneut empirisch zu belegen. Diese Herstellungsform bezeichnet Mbembe als „Verfahren des Fabulierens“. Indem der Zuschreibungsprozess ,,vielfach erfundene Tatsachen als real, sicher und exakt darstellte, umging er die Sache, die er zu erfassen vorgab, und nahm selbst dann ein zutiefst von Phantasien geprägtes Verhältnis zu ihr ein, wenn er den Anspruch auf eine objektive Darstellung erhob“ (Mbembe 2015, S. 31). Infolge der Zuschreibungspraktik werden Privilegien vergeben bzw. versagt und weitere soziale Zuschreibungen und Bewertungen (z. B. sog. ,,illegale“ oder „,irreguläre“ Migration) mit den (nun identifizierten) „Eigenschaften“ des Kollektivs wiederum korreliert (vgl. u. a. Munsch 2014, S. 69 f.). Im Anschluss an einen solchen Prozess der Identifizierung und Klassifizierung von Gruppen bliebe danach, ,in der Tat nur noch zu zeigen, wodurch sie sich voneinander unterscheiden“ (Mbembe 2015, S. 41).

Ohne genauer auf alle einzelnen Konsequenzen einzugehen, soll an dieser Stelle ein weiterer und bisher verdeckter Aspekt expliziert werden. In den oben skizzierten Definitionen von Migration war bisher nur implizit spürbar, dass migratorische Mobilität - in Form von „Wanderung“ oder ,dauerhaftem Wohnortswechsel“ - als thematischer Ausgangspunkt zur Bestimmung von Migration gewählt wird (vgl. Eichler 2008; Wagner 2008). Diese triviale Feststellung birgt enorme epistemologische Sprengkraft. Die Migrant_innen werden einseitig thematisiert, sie werden als außergewöhnlich hervorgebracht und als abweichend von der Norm(alität) der (imaginierten identitätslogisch konzipierten) Mehrheit gerahmt. Diese Besonderung bewertet damit die migratorische Mobilität negativ, verdeckt den Standpunkt der sesshaften Bezeichnenden und stuft wiederum deren Immobilität implizit als positiv ein. Danach wird migratorische Mobilität letztlich als „Ausnahmezustand“ (Agamben 2004) thematisch und repräsentiert in diesem Zusammenhang das Anomische im Gegensatz zu der vorausgesetzten, selbstverständlichen und unhinterfragten Normalität der Sesshaftigkeit (vgl. Canguilhem 1974).

\section{Migration als „Normalzustand“}

Die bisherige Latenz der Dimension der Sesshaftigkeit im Migrationsbegriff gibt einen möglichen Anhaltspunkt, weshalb Sesshaftigkeit im Kontext von Migrationsforschung kategorial irrelevant zu sein scheint (vgl. de Genova 2014, S. 224). Mit der Sensibilisierung der im Migrationsbegriff verdeckten Dimension der Sesshaftigkeit und Immobilität wird ihre Latenz aufgebrochen und ihr bisher verschwiegener „Normalzustand“ intelligibel. Mit der Kontingentsetzung wird ein Perspektivwechsel eingeleitet, der nun den Pol der Sesshaftigkeit mit einbezieht und so die „Möglichkeit von Alternativen“ (Marchart 2013, S. 8) sichtbar macht. In der bisher üblichen Begriffspraktik war es zentral, dass ausschließlich Migrant_innen und ihre Mobilität bezeichnet wurden, nicht aber Sesshafte in den Fokus gerieten. Inhetveen charakterisiert dies als ,,sedentaristischen Bias“ und sensibilisiert für die Folgen, die in der Aufrechterhaltung dieser Strategie implizit bleiben: „Wenn das Bleiben, anders als das Mobilsein, als normal angesehen wird, hat das nicht nur analytische, sondern 
nicht zuletzt politische Implikationen: Der Sedentarismus legitimiert Politiken der Abschottung" (Inhetveen 2010, S. 554).

Positionen, die insbesondere diese „Politiken der Abschottung" kritisieren, begreifen Migration aus einer nomadischen Perspektive, wonach ,Migration ein selbstverständlicher Bestandteil menschlicher Lebensweise [ist]" (Hamburger 2010, S. 882, Herv. i. O.), und betonen Migration als „Lebensform“ (Pries 2001, S. 5), die „so alt wie die Menschheit" (ebd.) und in allen Teilen der Welt anzutreffen sei (vgl. auch Gebhardt und Hitzler 2006). In seinem Essay Planet der Nomaden zeichnet Schlögel (2006) die migratorische Lebensform nach und stellt fest, dass das „moderne Nomadentum viele Gesichter [hat] und fast alle Sprachen der Welt [spricht]. Es findet sich überall, selbst in Provinzen, die weitab von den weltgeschichtlichen Schütterzonen liegen“ (ebd., S. 25). Mit Oltmer ist hinzuzufügen, dass „Migration seit jeher ein zentrales Element der Anpassung des Menschen an Umweltbedingungen und gesellschaftliche Herausforderungen [bildet]“ (Oltmer 2012, S. 8) und die „Vorstellung ein Mythos, räumliche Bevölkerungsbewegungen (...) seien erst eine Erscheinung der Moderne oder gar Gegenwart" (Oltmer 2015, S. 21). Gegenwärtige Diskurse, die Mobilität als besondere Ausnahme stilisieren, werden ebenso von Hahn kritisiert. Sie stellt fest, dass gleichgültig, wie ,weit man in der Geschichte der Menschheit auch zurückblickt, ob einige Jahre, Jahrzehnte oder sogar Jahrtausende, stets treffen wir auf Gesellschaften, auf Gruppen von Menschen, auf einzelne Individuen, die unterwegs waren, herumwanderten, migrierten“" (Hahn 2012, S. 193). Die Latenz der Dimension Sesshaftigkeit wirkt gerade aufgrund ihrer unbegriffenen Normativität gewaltsam, wie in den frames angedeutet wurde. Polemisch gewendet, rückt aus dieser Perspektive nun Sesshaftigkeit als begründungs- und legitimationsbedürftiger „Ausnahmezustand“ in den Fokus der Überlegungen zu Migration. In diesem Horizont bleibt in Anschluss an Schlögel - ,[s]obald die Idee der Seßhaftigkeit nicht mehr selbstverständlich ist" (Schlögel 2006, S. 38) - danach nicht die Frage, „warum [begeben sich] so viele Menschen auf Wanderschaft, sondern im Gegenteil: warum bleiben [sie], wo es doch so viele Gründe gibt, wegzugehen“ (ebd.).

\section{Zwischenfazit}

Fassen wir das Bisherige zusammen: In der Skizzierung der Momente der Sesshaftigkeit bzw. Immobilität und des Nomadentums bzw. der Mobilität im Migrationsbegriff wird deutlich, dass beide Positionen, sofern sie identitätslogisch konzipiert werden, in ihrer dichotomen Frontstellung nicht hinreichend das Phänomen Migration klären können. Mit der Problematisierung der bisher als zentral erkannten identitätslogischen Fassung von Migration in ihrer Spannung zwischen Mobilität und Sesshaftigkeit kann somit an dieser Stelle noch lange nicht Schluss sein. Beide Perspektiven auf Migration ,stellen zwar eine kritische Intervention im Hinblick auf wissenschaftliche Praxen defizitärer Zuschreibung und Verobjektivierung dar, zugleich aber wiederholen sie (freilich unter veränderten Vorzeichen) die Unterscheidung" (Mecheril et al. 2013, S. 18). Das bedeutet, dass die einfache Umkehrung innerhalb ihrer identiätslogischen und essentialistischen Grundorientierung niemals 
vollständig ihrem binären Grundschema zwischen Statik und Bewegung entkommen kann. Diese Verwicklung in ,definitorische Kasuistik“ (Brubaker 2007, S. 21) und die Erarbeitung weiterer ontologischer Merkmale trägt wenig dazu bei, den bisherigen Fallstricken zu entkommen (vgl. Mecheril 2008). Die Anreicherung durch Kontingenz trägt dementgegen zur Pluralisierung einer bislang vorherrschenden Naturalisierung möglicher Unterscheidungen bei und verdeutlicht die Leerstellen essentialistischer Zugänge (vgl. Nagel 2010, S. 603). Richtet man das Interesse erneut auf die angeführte Ausgangslage und nähert sich Migration auf einer kategorialen Ebene, d. h. werden die impliziten Voraussetzungen des bisher Dargelegten fokussiert und damit auch ,die Wissensbestände, die diese ermöglichen und stabilisieren“ (Mecheril et al. 2013, S. 35), verdeutlichen sich Perspektiven auf Migration, deren Diskussion für Migrationsforschung in der Erwachsenenbildung von Interesse sein könnten.

\subsection{Eine Verdeutlichung}

An einem aktuellen Beispiel soll dieser Zusammenhang erneut verdeutlicht werden. Die Expertise des Deutschen Instituts für Erwachsenenbildung (DIE) mit dem Titel Diversität und Weiterbildung in Bremen und Bremerhaven (Fleige et al. 2015)

soll Empfehlungen dazu liefern, wie Weiterbildungseinrichtungen in den Kommunen Bremen und Bremerhaven sich angesichts der kulturellen Vielfalt und Diversität der Gesellschaft interkulturell öffnen können und unter welchen Bedingungen dies gelingen kann (ebd., S. 3).

In diesem Zusammenhang stehen insbesondere „Menschen mit Migrationshintergrund“" und deren Beteiligung an Weiterbildung im Fokus. In der Expertise fällt auf, dass Aussagen zum Konstrukt „Mensch(en) mit Migrationshintergrund“ auf kategorialer Ebene nicht zu finden sind. Damit entsteht der Eindruck, dass diese Auseinandersetzung nicht notwendig zu sein scheint, weil bereits im Voraus hinreichend geklärt wurde, was „Menschen mit Migrationshintergrund“ auszeichnet. In der Identifizierung der Zielgruppe „Menschen mit Migrationshintergrund“ über Defizitbeschreibungen und sozialstatistische Markierungen (vgl. ebd., Fußnote 2) wird Migration danach identitätslogisch auf einen bestimmten (,exklusiven“ und prekären) Bereich verengt (vgl. Clam 2002, S. 21 f.). Fischer macht bei einer solchen (unbegründeten kategorialen) Übernahme des Konstrukts „Menschen mit Migrationshintergrund" deutlich, dass sie

lediglich als Sonder- bzw. Randgruppe betrachtet [werden], was einerseits ihrem Anteil an der Gesamtbevölkerung nicht mehr entspricht und andererseits Ignoranz gegenüber gesellschaftlichen Entwicklungen verrät, die zunehmend durch Prozesse der Globalisierung und Migration geprägt sind“ (Fischer 2011, S. 31).

Diese exkludierende Verdinglichung von Migration (fallacy of misplaced concreteness - Whitehead) adressiert damit jene Gefahren, die bereits oben hervorgehoben wurden. Das alltagsweltliche Verständnis von „Migrant_innen“ ist somit Bestandteil dessen, was geklärt werden will, und nicht dessen, womit erklärt werden will (vgl. 
Brubaker 2007, S. 19). Vor diesem Hintergrund drängt sich die Frage auf, ob ein solcher Zugang nicht eher dazu beiträgt, Schließungen hervorzubringen als zu einer Öffnung von „Diversität“ beizutragen. Damit scheint auf, wie die (nicht offengelegte) kategoriale Entscheidung wissenschaftlicher Forschung sich auf leisen Sohlen Einlass in die Untersuchung verschafft und als blinder Fleck Bestandteil dessen ist, was zu beschreiben getrachtet wurde. Denn mit der Anerkennung eines Perspektivwechsels auf Sesshaftigkeit könnten nun auch die (immobilen) „Einheimischen“ endlich als mögliche Adressat_innen spezifischer „Zielgruppen“von Weiterbildung in den Blick geraten und nicht ausschließlich „Migrant_innen“. Damit kämen zugleich die Relationen zwischen differenten Formen von Mobilität und transitiver Identitätsentwicklung als Lernanlass in den Blick und nicht mehr allein identifizierbare Personen (vgl. Schäffter 2014).

\subsection{Die gesellschaftliche Dimension kategorialer Arbeit}

An dieser Stelle könnten Einwände laut werden, was denn eine begriffliche Annäherung an den Migrationsbegriff letztlich zu leisten imstande wäre. „Eigentlich“ ändere sich doch ,nichts“ an der Situation von Migrant_innen, wenn begriffsanalytisch gearbeitet werde. Zudem scheint es doch offensichtlich zu sein, dass es eindeutig reale Migrant_innen gibt, die sich in ständigen und bisweilen existenziellen (Überlebens-)Kämpfen befinden. D. h., es gibt Interessenkonflikte in vielfältiger Hinsicht, die nicht ,wegargumentiert“" oder durch die Auflösung eines essentialistischen Zugangs vermieden werden können. Dahinter steht die naheliegende Frage und wohl auch Beklemmung, wie mit der Kategorie Migration umgegangen werden soll, wenn sie von ihrer bisherigen Essentialisierung und Hypostasierung entledigt wird. Werden damit nicht geradezu einer endlosen Arbitrarität und einem anything goes die Türen geöffnet? Hier tut sich gewissermaßen ein größeres Problem auf, dessen umfängliche Diskussion an dieser Stelle nicht geführt werden kann, sondern in weiteren Überlegungen anzugehen wäre. Erste Hinweise sollen skizziert werden:

Vor einem politischen Hintergrund ist es auch durchaus klar, dass normative Entscheidungen getroffen werden müssen, wenn politische Ziele erreicht und durchgesetzt werden wollen. Dafür wird ein Fundament benötigt, das geradezu als kontingentresistent aufgefasst werden muss, um Einigkeit (Identität) behalten zu können. Insbesondere für Migrant_innen und ihre Vertretungen ist es nur folgerichtig, diese Identität keinesfalls infrage zu stellen. Das liegt notwendigerweise auch daran, dass politisches Engagement ohne das Fundament einer identitätslogischen Fassung (wohl) unmöglich ist. Diese dilemmatische Situation, einerseits Migrant_innen als eine Gruppe mit gemeinsamen Eigenschaften und gemeinsamer Identität zu adressieren, bringt, wie oben gezeigt wurde, unüberwindbare Schwierigkeiten mit sich. Andererseits verliert migrationspolitisches Engagement jegliche Bedeutung, wenn ein ,identitärer Kern“ fehlt.

Um nicht missverstanden zu werden: Radikaler Protest und Mobilisierung sowie politische Forderungen von Migrant_innenorganisationen oder migrantischer Initiativen werden hier nicht in Abrede gestellt, sondern in ihrer Bedeutung für Empowerment betont (vgl. auch Fischer 2011, S. 23). Es geht darum, an diese Essentialisierungsstrategie - in dieser Hinsicht verstehe ich Heinemanns (2014, S. 18) 
Hinweis auf G. Spivaks Konzept des strategic essentialism - noch einen weiteren Gedanken anzuknüpfen, der sich unweigerlich einschleicht, sobald ontologisch auf eine identitätslogische Konzeptionalisierung von Migration zurückgegriffen wird. Sofern Migration identitätslogisch verdinglicht wird, zieht das unaufhörlich Diskurse nach sich, die den deskriptiven Gehalt des Begriffs herausfordern. Es müssen immer wieder neue und andere Eigenschaftsmerkmale angeboten, ausdifferenziert, modifiziert oder korrigiert werden, damit deutlich bleibt, wer dieser Gruppe angehört bzw. aus ihr ausgeschlossen ist. Dies hat zur Folge, dass es andauernd neue und weitere Aufspaltungen innerhalb der vermeintlich festen Identität geben wird, die die (benötigte) Einheit bedrohen. Paradoxerweise wird damit aber gerade dort das Ziel, die Herstellung einer Einheit, verfehlt, wo es zu treffen geglaubt wird. Damit kann eine identitätslogische Konzeption von Migration nicht als Ausgangspunkt gewählt werden, da sie niemals einen festen Grund abzugeben vermag, selbst wenn sie ihn noch so deutlich postuliert (vgl. u. a. Butler 1993). An dieser Stelle könnten alternative Überlegungen zur Erhellung der Problematik herangezogen werden, die anstelle der Stilllegung des Migrationsbegriffs seine dynamische Potenz in den Mittelpunkt stellen. In einem bildungstheoretischen Zusammenhang erscheint dabei das Konzept einer transitorischen Identität individueller Biografien des Übergangs vielversprechend (vgl. Straub und Renn 2002; von Felden et al. 2014).

\section{Anschließende Überlegungen}

Die hier vorgetragene Skizze eines ersten Zugangs zu einer kategorialen Annäherung an den Migrationsbegriff befindet sich sicherlich noch am Beginn weiterer erforderlicher Denkbewegungen. Mit der gewählten Herangehensweise sind einzelne Überlegungen in einen fragmentarischen Zusammenhang gerückt worden mit dem Ziel, alternative Perspektiven aufscheinen zu lassen (vgl. Jaeggi und Wesche 2013, S. 8). Daher wurde notwendigerweise darauf verzichtet, alle Bezüge einer ausführlicheren Diskussion zuzuführen. Es ginge jedoch vollständig an der hier vorgetragenen Position vorbei, wenn erwartet würde, ,Migration“ oder „Menschen mit Migrationshintergrund" auf einer inhaltlich substanziellen Ebene operationalisieren zu wollen. Vielmehr stellt der vorgetragene Argumentationsgang derartige Zuschreibungen bereits methodologisch auf einer kategorialen Ebene grundsätzlich und entschieden infrage. Dieses Vorgehen wird als epistemologische Begriffsklärung bei der wissenschaftlichen Gegenstandsbestimmung bezeichnet und sollte insbesondere bei einem gesellschaftspolitisch hochsensiblen Problembereich wissenschaftstheoretisch Berücksichtigung finden. Die Relevanz dieser Überlegungen für Erwachsenenbildung besteht daher in der Aufforderung, nicht länger hinter das methodologische Niveau kultur- und sozialwissenschaftlicher Forschungsansätze zurückzufallen und somit an die hier eingeführten Diskurse Anschluss zu finden.

Vor diesem Hintergrund soll der angestellte Streifzug vor allem dazu inspirieren, den Migrationsdiskursen in der Erwachsenenbildung einen weiteren Aspekt hinzuzufügen. Sofern es also gewagt wird, einen Schritt weiter zu gehen, kann der Einsatz einer dichotomen essentialistischen Gegenüberstellung von Sesshaftigkeit oder Nomadentum nur einen Zwischenhalt darstellen auf dem Weg, sich auf das 
komplementäre Verhältnis zwischen beiden Polen einzulassen. Folglich wird Migration nicht mehr identitätslogisch aufzufassen zu sein, sondern hinreichend nur als „Schauplatz ständiger Offenheit und Umdeutbarkeit“ (Butler 1993, S. 50) adressiert werden. Der (vermeintliche) Verlust von Eindeutigkeit ist demnach nur auf den ersten Blick bedauerlich. Die Umstellung auf eine differenzlogische Konzeptionalisierung von Migration ist mitnichten neu oder originell, sondern bereits Bestandteil etablierter Diskurse. Beispielsweise stellt Mecheril fest, dass wer „,eine Migrantin“ ist, ist nicht von vornherein festgelegt, sondern muss einerseits als diskursives Produkt, andererseits als Ergebnis kontextspezifischer und lokaler Praxen verstanden werden“ (Mecheril 2004, S. 47). Allerdings führte dieser Befund bisher nicht dazu, dass er sich vollständig in der Theoriebildung niederschlug.

In der Umstellung auf eine differenzlogische Rahmung wird Migration von ihren bisherigen Ontologien befreit und ,,bislang unvorhergesehene Bedeutungen [können] zum Tragen kommen“(Butler 1993, S. 50). Mit anderen Worten: Migrant_innen werden damit nicht mehr deskriptiv als Identität totalisiert, sondern es wird ermöglicht, dass ,kontingente Gründe immer wieder aufs Neue gefunden und gelegt werden müssen, auch wenn sie sich als noch so temporär, partiell und instabil erweisen sollten“ (Marchart 2013, S. 11). Dass dieser kategoriale Umbau nicht nebenbei erfolgt, ist einsichtig. Mit einer differenztheoretischen Herangehensweise dramatisiert sich dieser eingeschlagene Weg, weil die Stabilität der Kategorie nicht mehr vor$a b$ oder letztbegründet gegeben sein kann, sondern in immer wiederkehrenden und unaufhörlichen Auseinandersetzungen (re-)aktualisiert werden muss, was metaphorisch mehr an ein Manöver auf hoher See erinnert als an eines in einem sicher geglaubten Hafen. Als aussichtsreiche - um die Metaphorik ein weiteres Mal zu bemühen - Navigationsinstrumente, die eine identitätslogische Fassung von Migration suspendieren und weiterführende Überlegungen offerieren könnten, erscheinen Sartres (1980) Ansatz einer seriellen Kollektivität, Deleuzes (1992) Ausarbeitungen einer seriellen Soziologie, Latours (2007) Überlegungen zu einer Soziologie der Assoziationen, Brubakers (2007) Studie Ethnizität ohne Gruppen oder auch Schäffters (2015) Ansatz eines relationalen Feldes. So unterschiedlich die versammelten Kandidaten auch sein mögen, so inspirierend sind sie für die kategoriale Aufspannung von Migration außerhalb essentialistischer Engführungen, weil sie mit der entscheidenden theoriestrategischen Annahme - mit der Umstellung der Grundorientierung von Identität auf Differenz - arbeiten. Erst an dieser Stelle beginnt somit die ,eigentliche“ Herausforderung, vor der gegenwärtig offensichtlich noch zurückschreckt wird, vielleicht weil damit die eigene sicher geglaubte Position auch kontingent werden würde. Infolgedessen ist Migration keineswegs eine mittlerweile erforschte, sondern eine bedauerlicherweise erfolgreich stillgelegte Kategorie (vgl. Nuissl 2012). Wie wäre es wohl, die noch nicht ausgeschöpfte kategoriale Potenzialität erwachsenenpädagogischer Migrationsforschung entschiedener anzugehen? Sofern es also zutrifft, begriffsanalytische Arbeit in ihrer epistemischen und gesellschaftlichen Dimension ernst zu nehmen, wäre wohl nicht mehr zu fragen ,Was ist Migration? “, sondern „Wie, wann, warum und von wem wird Migration als fundamentale Analyseeinheit gedeutet und welche Konsequenzen beinhaltet diese Deutung für erwachsenenpädagogische Erkenntnisentwicklung?“، 
Open Access. Dieser Artikel wird unter der Creative Commons Namensnennung 4.0 International Lizenz (http://creativecommons.org/licenses/by/4.0/deed.de) veröffentlicht, welche die uneingeschränkte Nutzung, Verbreitung und Wiedergabe für beliebige Zwecke erlaubt, sofern Sie den/die ursprünglichen Autor(en) und die Quelle ordnungsgemäß nennen, einen Link zur Creative Commons Lizenz beifügen und angeben, ob Änderungen vorgenommen wurden.

\section{Literatur}

Agamben, G. (2004). Ausnahmezustand. Homo sacer II.1. Frankfurt a.M.: Suhrkamp.

Bachelard, G. (1980). Die Philosophie des Nein. Versuch einer Philosophie des neuen wissenschaftlichen Geistes. Frankfurt a.M.: Suhrkamp.

Bedorf, T., \& Röttgers, K. (Hrsg.). (2010). Das Politische und die Politik. Frankfurt a.M.: Suhrkamp.

Bilger, F. (2006). Migranten und Migrantinnen - eine weitgehend unbekannte Zielgruppe in der Weiterbildung. Empirische Erkenntnisse und methodische Herausforderungen. Report. Zeitschrift für Weiterbildungsforschung, 29(2), 21-31.

Bilger, F. (2011). Weiterbildungsverhalten von Personen mit Migrationshintergrund. Ein nach wie vor wenig erforschtes Feld. Hessische Blätter für Volksbildung, 61(4), 353-360.

Bilger, F., \& Hartmann, J. (2011). Weiterbildung von Personen mit Migrationshintergrund. In B.von Rosenbladt, \& F. Bilger (Hrsg.), Weiterbildungsbeteiligung 2010. Trendanalyse auf Basis des deutschen AES (S. 97-101). Bielefeld: W. Bertelsmann.

Brubaker, R. (2007). Ethnizität ohne Gruppen. In R. Brubaker (Hrsg.), Ethnizität ohne Gruppen (S. 16-45). Hamburg: Hamburger Edition.

Buckel, S., Georgi, F., Kannankulam, J., \& Wissel, J. (2014). Theorie, Methoden und Analysen kritischer Europaforschung. In Forschungsgruppe „Staatsprojekt Europa“ (Hrsg.), Kämpfe um Migrationspolitik. Theorie, Methode und Analysen kritischer Europaforschung (S. 15-86). Bielefeld: Transcript.

Bünger, C. (2015). Bildung - Macht - Subjektivierung? Sondierungen im Feld der Bildungsforschung. In E. Christof, \& E. Ribolits (Hrsg.), Bildung und Macht-eine kritische Bestandsaufnahme (S. 15-29). Wien: Löcker.

Butler, J. (1993). Kontingente Grundlagen: Der Feminismus und die Frage der „Postmoderne“. In S. Benhabib, J. Butler, D. Cornell, \& N. Fraser (Hrsg.), Der Streit um Differenz. Feminismus und Postmoderne in der Gegenwart (S. 31-58). Frankfurt a.M.: Fischer.

Butler, J. (2010). Raster des Krieges: Warum wir nicht jedes Leid beklagen. Frankfurt a.M.: Campus.

Canguilhem, G. (1974). Das Normale und das Pathologische. Frankfurt a.M.: Ullstein.

Castro Varela, M. M. (2013a). Ist Integration nötig? Eine Streitschrift. Ettenheim: Lambertus.

Castro Varela, M. M. (2013b). „Parallelgesellschaften“ und „Nationalmannschaften“ - Überlegungen zur Kritik in der Kritischen Migrationsforschung. In P. Mecheril, O. Thomas-Olalde, C. Melter, S. Arens, \& E. Romaner (Hrsg.), Migrationsforschung als Kritik? Konturen einer Forschungsperspektive (S. 65-78). Wiesbaden: VS Verlag für Sozialwissenschaften.

Clam, J. (2002). Was heißt: Sich an Differenz statt an Identität orientieren? Zur Deontologisierung in Philosophie und Sozialwissenschaft. Konstanz: UVK.

de Genova, N. (2014). Die queeren Politiken der Migration: Reflektionen über „Illegalität“ und Unverbesserlichkeit. In L.-M. Heimeshoff, S. Hess, S. Kron, H. Schwenken, \& M. Trzeciak (Hrsg.), Grenzregime II. Migration, Kontrolle, Wissen, Transnationale Perspektiven (S. 217-231). Berlin: Assoziation A.

Dietze, G. (2009). Okzidentalismuskritik. Möglichkeiten und Grenzen einer Forschungsperspektivierung. In D. Gabriele, C. Brunner, \& E. Wenzel (Hrsg.), Kritik des Okzidentalismus. Transdisziplinäre Beiträge zu (Neo-)Orientalismus und Geschlecht (S. 23-54). Bielefeld: Transcript.

Deleuze, G. (1992). Woran erkennt man den Strukturalismus. Berlin: Merve.

Deleuze, G., \& Guattari, F. (2000). Was ist Philosophie? Frankfurt a.M.: Suhrkamp.

Eichler, K. J. (2008). Migration, transnationale Lebenswelten und Gesundheit. Eine qualitative Studie über das Gesundheitshandeln von Migrantinnen. Wiesbaden: VS Verlag für Sozialwissenschaften.

Erler, I., Holzer, D., Kloyber, C., \& Ribolits, E. (Hrsg.). (2012). Kritisch denken: für eine andere Erwachsenenbildung. Innsbruck: Studienverlag.

Fischer, V. (2011). Erwachsenenbildung im Migrationskontext. In C. Zeuner (Hrsg.), Enzyklopädie Erziehungswissenschaft online (S. 1-35). Weinheim: Beltz/Juventa. DOI 10.3262/EEO16110203. Zugegriffen: 10. Nov. 2015 
Fleige, M., Zimmer, V., Lücker, L., \& Thom, S. (2015). Diversität und Weiterbildung in Bremen und Bremerhaven. https://ssl2.bremen.de/lernen_vor_ort/sixcms/media.php/13/Expertise_Diversitaet_ Weiterbildung.pdf. Zugegriffen: 10. Nov. 2015

Gebhardt, W., \& Hitzler, R. (Hrsg.). (2006). Nomaden, Flaneure, Vagabunden. Wissensformen und Denkstile der Gegenwart. Wiesbaden: VS Verlag für Sozialwissenschaften.

Han, P. (2000). Soziologie der Migration. Erklärungsmodelle - Fakten - Politische Konsequenzen - Perspektiven. Stuttgart: Lucius \& Lucius.

Han, P. (2006). Theorien zur internationalen Migration. Stuttgart: Lucius \& Lucius.

Hahn, S. (2012). Historische Migrationsforschung. Frankfurt a.M.: Campus.

Hamburger, F. (2010). Weiterbildung von Migranten. In R. Tippelt, \& A.von Hippel (Hrsg.), Handbuch Erwachsenenbildung/Weiterbildung (S. 881-888). Wiesbaden: VS Verlag für Sozialwissenschaften.

Hamburger, F., \& Stauf, E. (2009). „Migrationshintergrund“ zwischen Statistik und Stigma. Denkanstoß zu einem häufig verwendeten Begriff. Schüler. Wissen für Lehrer. Migration, 30-31.

Heinemann, A. (2014). Teilnahme an Weiterbildung in der Migrationsgesellschaft. Perspektiven deutscher Frauen mit „Migrationshintergrund“. Bielefeld: Transcript.

Heinemann, A., \& Robak, S. (2012). Interkulturelle Erwachsenenbildung. In H.-J. Roth, \& C. Anastasopoulos (Hrsg.), Enzyklopädie Erziehungswissenschaft online. Weinheim: Beltz/Juventa. DOI 10.3262/EEO06120239. Zugegriffen: 10. Nov. 2015

Heimeshoff, L.-M., Hess, S., Kron, S., Schwenken, H., \& Trzeciak, M. (Hrsg.). (2014). Grenzregime II. Migration, Kontrolle, Wissen, Transnationale Perspektiven. Berlin: Assoziation A.

Herrmann, G. (2014). Ein Mehr an Freiheit durch ein Mehr an Sicherheit? Zur Organisation von Zirkulation in der Europäischen Union: Die Beispiele des Grenz- und Migrationsmanagements. In A. Vasilache (Hrsg.), Gouvernementalität, Staat und Weltgesellschaft. Studien zum Regieren im Anschluss an Foucault (S. 137-170). Wiesbaden: Springer Fachmedien.

Hess, S., \& Kasparek, B. (2010). Grenzregime. Diskurse, Praktiken, Institutionen in Europa. Berlin: Assoziation A.

Holzer, D. (2009). Kritisch-emanzipatorische Erwachsenenbildung: totgesagt und doch lebendig? Magazin erwachsenenbildung.at, 7/8. www.erwachsenenbildung.at/magazin/09-7u8/meb09-7u8.pdf. Zugegriffen: 10. Nov. 2015

Inhetveen, K. (2010). In alle Himmelsrichtungen? Transnationale Migration in einer Welt konfliktreicher Mobilitäten. In H.-G. Soeffner (Hrsg.), Transnationale Gesellschaften (Bd. 2, S. 547-556). Wiesbaden: VS Verlag für Sozialwissenschaften.

Jaeggi, R., \& Karakayali, S. (2013). Einführung: Was ist Kritik? In R. Jaeggi, \& T. Wesche (Hrsg.), Was ist Kritik? (S. 7-22). Frankfurt a.M.: Suhrkamp.

Karakayali, S., \& Tsianos, V. (2007). Movements that matter. Eine Einleitung. In Transit Migration Forschungsgruppe (Hrsg.), Turbulente Ränder. Neue Perspektiven auf Migration an den Grenzen Europas (S. 7-22). Bielefeld: Transcript.

Klingovsky, U., \& Pawlewicz, S. (2014). Untiefen im Diskurs um das Lebenslange Lernen. Zur Transformation erwachsenenpädagogischer Handlungsformen. In D. Holzer, C. Kloyber, W. Schuster, \& S. Vater (Hrsg.), Wenn Weiterbildung die Antwort ist, was war die Frage? (S. 85-97). Innsbruck: Studienverlag.

Kursbuch (2015). 183 - Wohin flüchten?. Hamburg: Murmann.

Latour, B. (2007). Eine neue Soziologie für eine neue Gesellschaft. Frankfurt a.M.: Suhrkamp.

Lichtblau, K. (2011). Die Eigenart der kultur- und sozialwissenschaftlichen Begriffsbildung. Wiesbaden: VS Verlag für Sozialwissenschaften.

Marchart, O. (2010). Die politische Differenz. Berlin: Suhrkamp.

Marchart, O. (2013). Das unmögliche Objekt. Eine postfundamentalistische Theorie der Gesellschaft. Frankfurt a.M.: Suhrkamp.

Masschelein, J. (2003). Trivialisierung von Kritik. Kritische Erziehungswissenschaft weiterdenken. Zeitschrift für Pädagogik, 49(46), 124-141.

Masschelein, J., \& Ricken, N. (2002). Regulierung von Pluralität - Skizzen vom „Außen“. Erziehungsphilosophische Überlegungen zu Funktion und Aufgabe einer Allgemeinen Erziehungswissenschaft. Zeitschrift für Erziehungswissenschaft, Beiheft(1), 93-108.

Mbembe, A. (2015). Kritik der schwarzen Vernunft. Berlin: Suhrkamp.

McLaren, P. (1999). Kritische Erziehungswissenschaft im Zeitalter der Globalisierung. In H. Sünker, \& H.-H. Krüger (Hrsg.), Kritische Erziehungswissenschaft am Neubeginn?! (S. 10-34). Frankfurt a.M.: Suhrkamp.

Mecheril, P. (2004). Einführung in die Migrationspädagogik. Weinheim: Beltz. 
Mecheril, P. (2008). Anerkennung von Mehrfachzugehörigkeiten. Eine Leitlinie für Erwachsenenbildung in der Migrationsgesellschaft. Hessische Blätter für Volksbildung, 58(1), 41-49.

Mecheril, P. (2011). Wirklichkeit schaffen: Integration als Dispositiv. Aus Politik und Zeitgeschichte. 50 Jahre Anwerbeabkommen mit der Türkei, 43, 49-54.

Mecheril, P., Thomas-Olalde, O., Melter, C., Arens, S., \& Romaner, E. (2013). Migrationsforschung als Kritik? Erkundung eines epistemischen Anliegens in 57 Schritten. In P. Mecheril, O. Thomas-Olalde, C. Melter, S. Arens, \& E. Romaner (Hrsg.), Migrationsforschung als Kritik? Konturen einer Forschungsperspektive (S. 7-58). Wiesbaden: VS Verlag für Sozialwissenschaften.

Messerschmidt, A. (2014). Engagement mit Geschichtshintergrund. Zeitschrift des Informations- und Dokumentationszentrums Für Antirassismusarbeit in Nordrhein-Westfalen, 20(4), 8-12. www.ida-nrw. de/cms/upload/Ueberblick/Ueberblick_4_14.pdf. Zugegriffen: 10. Nov. 2015

Munsch, C. (2014). Wer sind eigentlich „Menschen mit Migrationshintergrund“? Über die Notwendigkeit eines reflexiven Migrationsbegriffs. Forum Erziehungshilfen, 20(2), 68-72.

Nagel, A.-K. (2010). Religionssoziologie in ,relationistischer“ Perspektive: Migration und religiöse Netzwerke. In H.-G. Soeffner (Hrsg.), Transnationale Gesellschaften (S. 603-616). Frankfurt a.M.: Campus.

Nuissl, E. (2012). Editorial. Report. Zeitschrift für Weiterbildungsforschung, 35(4), 7-8.

Oltmer, J. (2012). Globale Migration. Geschichte und Gegenwart. München: Beck.

Oltmer, J. (2015). Der lange Marsch. Europa im globalen Wanderungsgeschehen. Kursbuch 183 - Wohin flüchten? (S. 21-41). Hamburg: Murmann.

Öztürk, H. (2012). Soziokulturelle Determinanten der beruflichen Weiterbildungsbeteiligung von Erwachsenen mit Migrationshintergrund in Deutschland - Eine empirische Analyse mit den Daten des SOEP. Report. Zeitschrift für Weiterbildungsforschung, 35(4), 21-34.

Öztürk, H. (2014). Migration und Erwachsenenbildung. Bielefeld: W. Bertelsmann.

Öztürk, H., \& Reiter, S. (2015). Weiterbildung im Spiegel von Migranten. Stand und Perspektiven der Weiterbildungsforschung. DIE Zeitschrift für Erwachsenenbildung, 22(2), 31-34.

Pongratz, L. (2010). Kritische Erwachsenenbildung: Analysen und Anstöße. Wiesbaden: VS Verlag für Sozialwissenschaften.

Pries, L. (2001). Internationale Migration. Bielefeld: Transcript.

Rheinberger, H.-J. (2007). Historische Epistemologie. Eine Einführung. Hamburg: Junius.

Rocha, J. (2014). Die Bedingungen der Wissensproduktion über Migration. In L.-M. Heimeshoff, S. Hess, S. Kron, H. Schwenken, \& M. Trzeciak (Hrsg.), Grenzregime II. Migration, Kontrolle, Wissen, Transnationale Perspektiven (S. 141-151). Berlin: Assoziation A.

Sandkühler, H.J. (1990). Epistemologischer Materialismus und Dialektik. Zur Onto-Epistemologie ideeller Identitäten. In G. Pasternack (Hrsg.), Philosophie und Wissenschaften. Zum Verhältnis von ontologischen, epistemologischen und methodologischen Voraussetzungen der Einzelwissenschaften (S. 223-240). Frankfurt a.M.: Lang.

Sartre, J.-P. (1980). Kritik der dialektischen Vernunft. 1. Bd.: Theorie der gesellschaftlichen Praxis. Reinbek: Rowohlt.

Schäfer, A., \& Wimmer, M. (Hrsg.). (2004). Tradition und Kontingenz. Münster: Waxmann.

Schäffter, O. (2011). Die Kontingenzperspektive auf den Forschungsgegenstand. In C. Hof, J. Ludwig, \& B. Schäffer (Hrsg.), Steuerung - Regulation - Gestaltung. Governance-Prozesse in der Erwachsenenbildung zwischen Struktur und Handlung (S. 232-239). Baltmannsweiler: Schneider.

Schäffter, O. (2014). Relationale Zielgruppenbestimmung als Planungsprinzip. Zugangswege zur Erwachsenenbildung im gesellschaftlichen Strukturwandel. Ulm: Klemm und Oelschläger.

Schäffter, O. (2015). Die Kategorie der Relation - der paradigmatische Kern und einzelwissenschaftliche Anwendungsfelder. Working paper. https://www.erziehungswissenschaften.hu-berlin.de/de/ebwb/ team/schaeffter/katrel_16_1. Zugegriffen: 10. Nov. 2015

Schmidt, B., \& Tippelt, R. (2006). Bildungsberatung für Migrantinnen und Migranten. Report. Zeitschrift für Weiterbildungsforschung, 29(2), 32-42.

Schlögel, K. (2006). Planet der Nomaden. Pilsen: wjs-Verlag.

Schrader, J. (2015). Vorsätze. DIE Zeitschrift für Erwachsenenbildung, 22(3), 3.

Sprung, A. (2008). Man lernt nie aus? MigrantInnen in der Weiterbildung am Beispiel Österreichs bildungsforschung, 5(1). http://www.pedocs.de/volltexte/2014/4609/pdf/bf_2008_1_Sprung_ Migrantinnen_Weiterbildung.pdf. Zugegriffen: 10. Nov. 2015

Sprung, A. (2011). Zwischen Diskriminierung und Anerkennung. Weiterbildung in der Migrationsgesellschaft. Münster: Waxmann.

Sprung, A. (2012). Migration bewegt die Weiterbildung (?). Entwicklung, Trends und Perspektiven in Wissenschaft und Praxis. Report. Zeitschrift für Weiterbildungsforschung, 35(4), 11-20. 
Sprung, A. (2015). Fachkräfte mit Migrationsgeschichte. Migrationsbedingte Vielfalt des Personals in der Erwachsenenbildung. DIE Zeitschrift für Erwachsenenbildung, 22(3), 46-48.

Straub, J., \& Renn, J. (Hrsg.). (2002). Transitorische Identität. Der Prozesscharakter des modernen Selbst. Frankfurt a.M.: Campus.

von Felden, H., Schäffter, O., \& Schicke, H. (Hrsg.). (2014). Denken in Übergängen. Weiterbildung in transitorischen Lebenslagen. Wiesbaden: VS Verlag für Sozialwissenschaften.

Wagner, F. (2008). Nation-state Unbound? Die migrationstheoretische Herausforderung. In J. Wissel, \& S. Wöhl (Hrsg.), Staatstheorie vor neuen Herausforderungen. Analyse und Kritik (S. 87-105). Münster: Westfälisches Dampfboot.

Wimmer, A., \& Glick Schiller, N. (2003). Methodological Nationalism, the Social Sciences, and the Study of Migration: An Essay in Historical Epistemology. International Migration Review, 37(3), 576-610.

Zetter, R. (2015). Angstgetrieben. Wie die Furcht vor dem Fremden die europäische Einwanderungspolitik bestimmt. Kursbuch 183 - Wohin flüchten? (S. 42-59). Hamburg: Murmann. 